\title{
SUBORDINATE SEMIGROUPS AND ORDER PROPERTIES
}

\author{
AKITAKA KISHIMOTO* and DEREK W. ROBINSON
}

(Received 17 April 1980)

Communicated by J. B. Miller

\begin{abstract}
Let $S_{t}=\exp \{-t H\}, T_{t}=\exp \{-t K\}$, be $C_{0}$-semigroups on a Banach space $\mathscr{X}$. For appropriate $f$ one can define subordinate semigroups $S_{t}^{f}=\exp \{-t f(H)\}, T_{t}^{f}=\exp \{-t f(K)\}$, on $\mathcal{X}$ and examine order properties of the pairs $S, T$, and $S^{f}, T^{f}$. If $\mathcal{X}=L^{P}(X ; d v)$ we define $S_{t}>T_{t} \geqslant 0$ if $S_{t}-T_{t}$ and $T_{t}$ map non-negative functions into non-negative functions. Then for $p$ fixed in the range $1<p<\infty$ we characterize the functions for which $S_{t} \geqslant T_{1} \geqslant 0$ implies $S_{l}^{f}>T_{i}^{f}>0$ for each $L^{p}(X ; d v)$ and the converse is true for all $L^{P}(X ; d \nu)$. Further we give irreducibility criteria for the strict ordering of holomorphic semigroups. This extends earlier results for $L^{2}$-spaces.
\end{abstract}

1980 Mathematics subject classification (Amer. Math. Soc.): primary 47 D 05; secondary 46 E 30, 43 A 32.

\section{Introduction}

In two previous papers (Bratteli, Kishimoto and Robinson (1980); Kishimoto and Robinson (1980)), which we refer to as I and II, we analyzed order properties of self-adjoint contraction semigroups on Hilbert space. In this paper we extend our results to holomorphic contraction semigroups on $L^{p}$-spaces. The principal new method used in this generalization is a construction by Phillips (Phillips (1952)) of subordinate semigroups on Banach space.

First recall that a function $f$ on $(0, \infty)$ is called completely monotone if $f \in C^{\infty}(0, \infty)$ and

$$
(-1)^{n} f^{(n)}(x)>0, \quad x \in(0, \infty), n=0,1,2, \ldots
$$

Next we denote by $\mathfrak{T}_{0}$ the class of non-negative functions on $[0, \infty)$ which are continuous from the right at zero, differentiable on $(0, \infty)$, and such that $f^{\prime}$ is

- Copyright Australian Mathematical Society 1981

*n leave of absence from Yokohama City University. 
completely monotone on $(0, \infty)$. Further we denote by $\mathscr{R}$ the class of nonnegative monotone increasing functions on $[0, \infty)$ which are differentiable on $(0, \infty)$ and such that $f^{\prime}$ is completely monotone on $(0, \infty)$. Thus if $f \in \mathfrak{N}$ one has $0<f(0) \leqslant f(0+)$.

In I we characterized $\mathfrak{N}$ as the class of functions which respect the order properties of all pairs of self-adjoint contraction semigroups. The more restricted class $\mathfrak{N}_{0}$ had previously been examined by Bochner (Bochner (1955)) and Phillips (Phillips (1952)) in the context of semigroups on Banach space.

If $S_{t}=\exp \{-t H\}$ is a $C_{0}$-semigroup on a Banach space $\mathcal{X}$ and $f \in \mathscr{R}_{0}$ one can construct an operator $f(H)$ by standard methods of functional analysis for generators. (see Hille and Phillips (1957) Chapters XV and XXIII; Nelson (1958)). It then follows that $f$ generates a $C_{0}$-semigroup $S^{f}$. In fact Phillips (Phillips (1952)) constructed the semigroup $S^{f}$ by an ansatz of the form

$$
S_{t}^{f}=\int_{0}^{\infty} d \mu_{f}^{t}(\tau) S_{\tau}
$$

where $\mu_{f}^{t}$ is a family of positive measures and proved that the generator of $S^{f}$ extends $f(H)$. Nelson (Nelson (1958)) subsequently proved that $f(H)$ generates $S^{f}$.

The relation (*) is the key to the generalization of our previous $L^{2}$ analysis to $L^{p}$-spaces. The semigroups $S^{f}$ constructed in this fashion had been previously studied in a more special context by Bochner (Bochner (1955)) who refers to them as subordinate semigroups. We begin with a brief survey of the construction and characterization of these semigroups.

\section{Completely monotone maps}

The functions $f \in \mathscr{R}_{0}$ are central to Phillips construction of subordinate semigroups and they are closely related to the completely monotone maps of Bochner (Bochner (1955)). A continuous mapping $f ; x \in(0, \infty) \rightarrow f(x) \in(0, \infty)$ is called a completely monotone map if for every completely monotone function $g$ on $(0, \infty)$ the composite function $g \circ f$ is completely monotone. (Such mappings have more recently been called Bernstein functions (Berg and Forst (1975)).)

The following characterization of completely monotone maps is a synthesis of results of Bochner (Bochner (1955)), Schoenberg (Schoenberg (1938)) and Phillips (Phillips (1952)).

Proposition 1. Let $f$ be a positive function on $(0, \infty)$. The following conditions are equivalent.

1. $x \in(0, \infty) \rightarrow f(x) \in(0, \infty)$ is a completely monotone map. 
2. $f \in C^{\infty}(0, \infty)$ and $f^{\prime}$ is a completely monotone function.

3. $f(0+)$ exists and $f$ has a representation

$$
f(x)=f(0+)+\alpha_{f} x+\int_{0+}^{\infty} d \mu_{f}(t)\left(1-e^{-t x}\right)
$$

where $\alpha_{f}>0$ and $\mu_{f}$ is a positive measure such that

$$
\int_{0+}^{1} d \mu_{f}(t) t<+\infty, \quad \int_{1}^{\infty} d \mu_{f}(t)<+\infty .
$$

4. $f \in C^{\infty}(0, \infty)$ and $\exp \{-t f\}$ is a completely monotone function for all $t>0$.

5. $\exp \{-t f\}$ has a representation

$$
e^{-t f(x)}=\int_{0}^{\infty} d \mu_{f}^{t}(\tau) e^{-\tau x}, \quad t>0,
$$

where $t \rightarrow \mu_{f}^{t}$ is a continuous family of positive measures satisfying

a. $\mu_{f}^{s+t}(\tau)=\int_{0}^{\tau} \mu_{f}^{s}(\tau-\sigma) d \mu_{f}^{t}(\sigma)$,

b. $\int_{0}^{\infty} d \mu_{f}^{t}(\tau)<1$,

c. $\lim _{t \rightarrow 0+} \int_{\tau_{0}}^{\infty} d \mu_{f}^{t}(\tau)=0, \tau_{0}>0$.

The equivalence of Conditions 1, 2, 3, and 4 is established in Chapter 4 of Bochner's book (Bochner (1955)). The equivalence of Conditions 1 and 5 was established by Phillips (Phillips (1952)) through adaptation of the proof of the Levy-Khinchine formula.

In fact the equivalence of 1,2 , and 4 is an easy consequence of Leibniz's formula and an inductive argument. The equivalence of 2 and 3 is an integrated version of Bernstein's theorem on completely monotone functions (see Berg and Forst (1975)) and the equivalence of Conditions 4 and 5 is also an easy consequence of the same theorem. (This observation simplifies considerably the proof of Phillips.) For example if Condition 5 is valid

$$
\begin{aligned}
(-1)^{n} \frac{d^{n}}{d x^{n}} e^{-t f(x)} & =\int_{0}^{\infty} d \mu_{f}^{t}(\tau)(-1)^{n} \frac{d^{n}}{d x^{n}} e^{-\tau x} \\
& =\int_{0}^{\infty} d \mu_{f}^{t}(\tau)^{n} e^{-\tau x} \geqslant 0 .
\end{aligned}
$$

Conversely if 4 is valid Bernstein's theorem establishes the existence of a family $\mu_{f}^{\prime}$ of positive measures such that

$$
e^{-t f(x)}=\int_{0}^{\infty} d \mu_{f}^{t}(\tau) e^{-\tau x}
$$

Substituting this representation into the relation

$$
e^{-s f(x)} e^{-t f(x)}=e^{-(s+t) f(x)}
$$


and using the uniqueness of the Laplace transform one finds 5a. Condition $5 b$ follows by monotone convergence because

$$
1>e^{-t f(x)}=\int_{0}^{\infty} d \mu_{f}^{t}(\tau) e^{-\tau x}
$$

(More precisely one has

$$
e^{-t f(0+)}=\int_{0}^{\infty} d \mu_{f}^{t}(\tau)
$$

Finally

$$
\begin{aligned}
1-e^{-t f\left(1 / \tau_{0}\right)} & \geqslant \int_{0}^{\infty} d \mu_{f}^{t}(\tau)\left(1-e^{-\tau / \tau_{0}}\right) \\
& \geqslant \int_{\tau_{0}}^{\infty} d \mu_{f}^{t}(\tau)\left(1-e^{-\tau / \tau_{0}}\right) \\
& \geqslant\left(1-e^{-1}\right) \int_{\tau_{0}}^{\infty} d \mu_{f}^{t}(\tau)>0
\end{aligned}
$$

and $5 \mathrm{c}$ follows immediately.

Proposition 1 shows that the class $\mathfrak{T}_{0}$ consists of the continuous extensions of the completely monotone maps to $[0, \infty)$.

Note that if $f_{1}$ and $f_{2}$ are completely monotone maps then it follows by definition that $f_{1} \circ f_{2}$ is also a completely monotone map.

REMARK. There is also a resolvent characterization of completely monotone maps. By Leibniz's formula and Conditions 2 and 4 of Proposition 1 one readily establishes that $f$ is a completely monotone map if, and only if, $(\lambda+f)^{-1}$ is a completely monotone function for all $\lambda>0$. But by Laplace transformation of Condition 5 of Proposition 1 this is the case if, and only if $(\lambda+f)^{-1}$ has a representation

$$
(\lambda+f(x))^{-1}=\int_{0}^{\infty} d v_{f}^{\lambda}(\tau) e^{-\tau x}
$$

where the positive measures $\nu_{f}^{\lambda}$ now satisfy resolvent equations,

$$
(\mu-\lambda)^{-1}\left(\nu_{f}^{\mu}(\rho)-\nu_{f}^{\lambda}(\rho)\right)+\int_{0}^{\rho} \nu_{f}^{\lambda}(\rho-\sigma) d \nu_{f}^{\mu}(\sigma)=0 .
$$

\section{Subordinate semigroups}

If $S_{t}=\exp \{-t H\}$ is a $C_{0}$-semigroup of contractions on a Banach space $\mathscr{X}$ and $f \in \mathscr{N}$ we wish to define the subordinate semigroup $S^{f}$ with generator $f(H)$. For $f \in \mathfrak{T}_{0}$ this construction has been made by Phillips for quite general $C_{0}$-semigroups. 
Proposition 2. Let $S_{t}=\exp \{-t H\}$ be a $C_{0}$-semigroup of contractions on a Banach space $\mathcal{X}$ and take $f \in \mathfrak{T}_{0}$. Define $f(H)$ on $D(H)$, the domain of $H$, by the strong integral

$$
f(H)=f(0+) 1+\alpha_{f} H+\int_{0+}^{\infty} d \mu_{f}(\tau)\left(1-S_{\tau}\right) .
$$

If $\alpha_{f} \neq 0$ then $f(H)$ is closed and generates a $C_{0^{-}}$semigroup of contractions $S^{f}$.

If $\alpha_{f}=0$ then $f(H)$ is closable and its closure $\overline{f(H)}$ generates a $C_{0}$-semigroup of contractions $S^{f}$.

In both cases one has

$$
S_{t}^{f}=\int_{0}^{\infty} d \mu_{f}^{t}(\tau) S_{\tau}
$$

This proposition is essentially a combination of results of Phillips (Phillips (1952)) and (Nelson (1958)). Note that if $A \in D(H)$ one has

$$
\left(1-S_{t}\right) A=\int_{0}^{t} d s H S_{s} A
$$

and the strong integral

$$
I(H) A=\int_{0+}^{\infty} d \mu_{f}(t)\left(1-S_{t}\right) A
$$

is well defined. In fact

$$
\|I(H) A\|<\int_{0+}^{\delta} d \mu_{f}(t) t\|H A\|+2 \int_{\delta}^{\infty} d \mu_{f}(t)\|A\|,
$$

that is $I(H)$ is $H$-bounded with relative bound 0 . Thus the first statement follows by perturbation theory. The remainder of the proof is in Nelson (1958).

Remark. Suppose in Proposition 2 that $S$ is a holomorphic semigroup. It is natural to try to characterize the $f$ for which $S^{f}$ is also holomorphic. For this it is relevant to note that $f$ maps each cone $\left\{z ; z=r e^{i \theta},|\theta|<\alpha<\pi / 2\right\}$ into itself.

Next consider the extension of Proposition 2 to the larger class of functions $\Re$.

Each $f \in \mathscr{R}$ has a decomposition $f=f_{0}+f_{1}$, where

$$
f_{1}(0)=0 \quad f_{1}(x)=f(0+)-f(0), \quad x>0,
$$

and $f_{0}=f-f_{1} \in \mathfrak{M}_{0}$. Thus it is first necessary to give an appropriate definition of $f_{1}(H)$. In many cases this can be achieved by limiting procedures.

If $S_{t}=\exp (-t H\}$ is a self-adjoint semigroup of contractions on a Hilbert space $\mathcal{H}$ the usual functional analysis of operators gives

$$
f_{1}(H)=(f(0+)-f(0))(1-P)
$$


where $P$ is the unique self-adjoint projection onto the subspace of $\mathscr{K}$ invariant under $S$. For more general semigroups no such unique projection exists. (Even in the Hilbert space case $P$ is not unique unless one requires self-adjointness.) If, however, $S$ and $\mathcal{X}$ are such that the mean ergodic theorem is valid this can be used to define $f_{1}(H)$. If, for example, the limits

$$
P A=\lim _{T \rightarrow \infty} \frac{1}{T} \int_{0}^{T} d s S_{t} A
$$

exist for all $A \in \mathcal{X}$ one can define $f_{1}(H)$ by

$$
f_{1}(H) A=(f(0+)-f(0)) \lim _{T \rightarrow \infty} \frac{1}{T} \int_{0}^{T} d t\left(1-S_{t}\right) A .
$$

Of course weaker forms of the mean ergodic theorem could be used but each such form expresses $f_{1}(H)$ as a limit of convex combinations of $1-S_{t}$, that is as limits of bounded operators of the type covered by Proposition 2.

If $\mathcal{X}$ is uniformly convex the mean ergodic theorem is valid in the above form. In particular, this is the case if $\mathscr{X}=L^{p}(X ; d \nu)$ with $1<p<\infty$.

Definition 3. Let $S$ be a $C_{0^{-}}$-semigroup of contractions on a uniformly convex Banach space $\mathcal{X}$ and let $f \in \mathfrak{R}$. For each $T>0$ define $f_{T} \in \mathfrak{T}_{0}$ by

$$
\begin{aligned}
f_{T}(x)= & f(0)+\alpha_{f} x+\int_{0+}^{\infty} d \mu_{f}(t)\left(1-e^{-t x}\right) \\
& +(f(0+)-f(0)) \frac{1}{T} \int_{0}^{T} d t\left(1-e^{-t x}\right) .
\end{aligned}
$$

The subordinate semigroup $S^{f}$ is then defined by

$$
S_{t}^{f}=\lim _{T \rightarrow \infty} S_{t}^{f_{T}}
$$

where $S^{f_{T}}$ is defined by Proposition 2 and the strong limit exists by the mean ergodic theorem.

The Phillips representation

$$
S_{t}^{f}=\int_{0}^{\infty} d \mu_{f}^{t}(\tau) S_{\tau}
$$

given in Proposition 2 is particularly useful for the analysis of the stability of order properties. For example if $\mathcal{K}$ is any strongly closed convex cone in $\mathfrak{X}$ which is invariant under $S$ then it is automatically invariant under $S^{f}$. Instead of attempting to partially analyze these stability propertis for general $\mathcal{X}$ we next give a full analysis for $\mathcal{X}=L^{p}(X ; d \nu)$ with $1<p<\infty$. The discussion could be extended to $L^{\infty}(X ; d \nu)$ but this is simplified by the fact that every $C_{0}$-semigroup of positivity preserving transformations on $L^{\infty}(X ; d \nu)$ is uniformly continuous (see Appendix). 


\section{3. $L^{p}$-Spaces}

Let $\mathcal{X}=L^{p}(X ; d \nu)$ with $1<p<\infty$. The dual $\mathcal{X}$ of $\mathcal{X}$ is $L^{q}(X ; d \nu)$ with $q^{-1}+p^{-1}=1$. We denote by $X_{+}$and $X_{+}^{*}$ the ( $\nu$-almost everywhere) non-

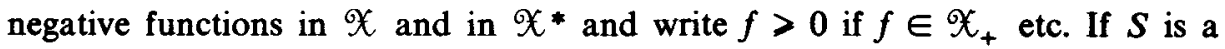
semigroup and $s_{t}$ leaves $\mathfrak{X}_{+}$invariant, that is if $S_{t} \mathfrak{X}_{+} \subseteq \mathfrak{X}_{+}$we use the notation of I and write $S_{t} \geqslant 0$. Thus $S_{t} \geqslant 0$ if, and only if,

$$
\omega\left(S_{t} f\right)>0
$$

for all $\omega \in \mathfrak{X}_{+}^{*}, f \in \mathfrak{X}_{+}$. Similarly we define $S_{t}>0$ if, and only if,

$$
\omega\left(S_{\downarrow} f\right)>0
$$

for all non-zero $\omega \in \mathcal{X}_{+}^{*}$ and $f \in \mathcal{X}_{+}$. Thus we can introduce an order relation between pairs of semigroups $S, T$, on $\mathcal{X}$ by setting $S_{t} \geqslant T_{t}$ if

$$
\omega\left(S_{\imath} f\right)>\omega(T, f)
$$

for all $\omega \in \mathcal{X}_{+}^{*}, f \in \mathscr{X}_{+}$. We can similarly define the strict order $S_{t}>T_{t}$.

Proposition 4. Let $S, T$, be $C_{0}$-semigroups of contractions on $\mathcal{X}=L^{p}(X ; d \nu)$ where $1<p<\infty$ and let $f \in \mathfrak{N}$ (or for $p=1$ let $f \in \mathscr{R}_{0}$ ).

If $S_{t} \geqslant 0$ for all $t>0$ then $S_{t}^{f} \geqslant 0$ for all $t>0$ and if $S_{t} \geqslant T_{t}$ for all $t>0$ then $S_{t}^{f} \geqslant T_{i}^{f}$ for all $t>0$.

Proof. Suppose $f_{0} \in \mathscr{M}_{0}$ and $\omega \in \mathscr{X}_{+}^{*}, g \in \mathscr{X}_{+}$, then

$$
\omega\left(S_{t}^{f_{g}} g\right)=\int_{0}^{\infty} d \mu_{f_{0}}^{t}(\tau) \omega\left(S_{\tau} g\right)>0 .
$$

Similarly

$$
\omega\left(\left(S_{t}^{f_{0}}-T_{t}^{f_{0}}\right) g\right)=\int_{0}^{\infty} d \mu_{f_{0}}^{t}(\tau) \omega\left(\left(S_{\tau}-T_{\tau}\right) g\right)>0 .
$$

This proves the result for $f_{0}$.

Now a general $f \in \mathscr{K}$ can be uniquely decomposed as $f=f_{0}+f_{1}$ where $f_{0} \in \Re_{0}$ and $f_{1}(0)=0, f_{1}(x)=f(0+)-f(0)$ for $x>0$. By Definition 3 one has $S^{f}=S^{f_{0}} S^{f_{1}}$ and

$$
S^{f_{1}}=\lim _{T \rightarrow \infty} S^{f_{T}}
$$

where

$$
S_{t}^{f_{T}}=e^{-c t} \exp \left\{\frac{c t}{T} \int_{0}^{T} d s S_{s}\right\}
$$

and $c=f(0+)-f(0)$. Therefore $S_{t} \geqslant 0$ implies that $S_{t}^{f} \geqslant e^{-c t} 1$ and hence

$$
S_{t}^{f}=\lim _{T \rightarrow \infty} S_{t}^{f_{0}} S_{t}^{f_{r}} \geqslant e^{-c t} S_{t}^{f_{0}} \geqslant 0 \text {. }
$$


Subsequently we will strengthen Proposition 4 to a result concerning the strict ordering but first we examine a converse in the spirit of $I$.

Consider the family $\mathscr{X}_{n}^{p} n=1,2,3, \ldots$, of finite-dimensional $L^{p}$-spaces with $p$ fixed and $1 \leqslant p \leqslant \infty$. (The index $n$ denotes the dimension.) Each $C_{0}$-semigroup on $\mathfrak{X}_{n}^{p}$ is of the form $S_{t}=\exp \{-t H\}$ where $H$ is an $n \times n$ matrix. The semigroup is real symmetric whenever $H$ is real symmetric. For such a semigroup to be contractive it is clearly necessary that $H>0$ and then Beurling and Deny, (Beurling and Deny (1958)), have shown that it is sufficient that

$$
H_{i j} \leqslant 0, \quad i \neq j ; \quad \sum_{j=1}^{n} H_{i j} \geqslant 0 .
$$

Now for each such contraction semigroup $S$ and each non-negative function $f$ on $[0, \infty)$ one can define $S^{f}$ by the usual matrix calculus. Explicitly if $E_{i}$, $i=1,2, \ldots, k$, denote the spectral projectors of $H$ and $\lambda_{i}$ the corresponding eigenvalues one has

$$
S_{t}^{f}=\sum_{i=1}^{k} e^{-t f\left(\lambda_{i}\right)} E_{i}
$$

Hence by Proposition 1, part 5,

$$
S_{f}^{f}=\sum_{i=1}^{k} \int_{0}^{\infty} d \mu_{f}^{t}(\tau) e^{-\tau \lambda} E_{i}=\int^{\infty} d \mu_{f}^{t}(\tau) S_{\tau}
$$

that is the spectral definition of $S^{f}$ and the abstract definition of Section 2 coincide.

The following result can now be deduced by combining Proposition 4 with the matrix calculations in I.

THEOREM 5. Let $f$ be a non-negative function on $[0, \infty)$ and suppose $1<p<$ $\infty$.

The following conditions are equivalent

1. $e^{-t H} \geqslant e^{-t K} \geqslant 0, t>0$, implies

$$
e^{-t f(H)} \geqslant e^{-t f(K)} \geqslant 0
$$

for all pairs of real symmetric contraction semigroups $\exp \{-t H\}, \exp \{-t K\}$ on the spaces $\mathfrak{X}_{n}^{p}, n=1,2,3, \ldots$

2. $f \in \mathfrak{N}$.

Proof. $2 \Rightarrow 1$ by Proposition 4 and $1 \Rightarrow 2$ by Theorem 7 of I. (Note that the proof of this Theorem 7 relies upon the special choice of matrices $H^{\prime}, K^{\prime}$ constructed from $H$ and $K$. These matrices depend upon a parameter $\theta$ and for $\theta$ 
sufficiently small and positive one has $H^{\prime} \geqslant 0, K^{\prime} \geqslant 0$, and the Beurling-Deny criterion for contractivity of the semigroups $S_{t}=\exp \left\{-t H^{\prime}\right\}, T_{t}=\exp \left\{-t K^{\prime}\right\}$, are satisfied. Thus the conclusion of Theorem 7 of $I$ is valid under the present more restrictive hypothesis.)

Next we examine the strict ordering relation. We begin with an analogue of Proposition 4.

Proposition 6. Let $S, T$, be $C_{0}$-semigroups of contractions on $\mathcal{X}=L^{p}(X ; d \nu)$ where $1<p<\infty$ and let $f \in \Re$ (or $p=1$ and $\left.f \in \Re_{0}\right)$. Assume that $f$ is not constant on some non-empty sub-interval of $(0, \infty)$.

If $S_{t}>0$ for all $t>0$ then $S_{t}^{f}>0$ for all $t>0$ and if $S_{t}>T_{t} \geqslant 0$ for all $t>0$ then $S_{t}^{f}>T_{t}^{f} \geqslant 0$ for all $t>0$.

Proof. Again consider the decomposition $f=f_{0}+f_{1}$ where $f_{0} \in \mathfrak{R}_{0}$ and $f_{1}$ is constant on $(0, \infty)$. Since $f$ is not constant on this interval one must have $f_{0}$ not constant. Consequently there exists a measurable subset $\Delta \subset(0, \infty)$ such that $\mu_{f_{0}}^{t}(\Delta)>0$. Thus if $S_{t}>0$ for all $t>0$ one must have

$$
\omega\left(S_{t}^{f} g\right)=\int_{0}^{\infty} d \mu_{f_{0}}^{f}(\tau) \omega\left(S_{\tau} g\right)>0
$$

for non-zero $\omega \in \mathcal{X}_{+}^{*}, g \in \mathfrak{X}_{+}$, that is $S_{t}^{f_{0}}>0$. Similarly $S_{t}>T_{t}$ for all $t>0$ implies $S_{t}^{f_{0}}>T_{t}^{f_{0}}$. This proves the proposition for $f_{0} \in \mathscr{T}_{0}$.

Next remark that

$$
e^{x \int_{0}^{T} d \tau S_{\tau}}-e^{x \int_{0}^{T} d \tau T_{\tau}}=x \int_{0}^{1} d \lambda e^{\lambda x \int_{0}^{T} d \tau S_{\tau}}\left(\int_{0}^{T} d s\left(S_{s}-T_{s}\right)\right) e^{(1-\lambda) x \int_{0}^{T} d \tau T_{r}} \geqslant 0
$$

for all $x>0$. Here we use $S_{s} \geqslant T_{s}$ and $T_{s} \geqslant 0$. But then

$$
\begin{aligned}
S_{t}^{f_{1}} & =\lim _{T \rightarrow \infty} e^{-c \frac{1}{T} \int_{0}^{T} d \tau\left(1-S_{r}\right)} \\
& \geqslant \lim _{T \rightarrow \infty} e^{-c_{T}^{\frac{1}{T}} \int_{0}^{T} d \tau\left(\mathbf{1}-T_{r}\right)}=T_{t}^{f_{1}} \geqslant e^{-c t} \mathbf{1}
\end{aligned}
$$

where $c=f(0+)-f(0)$. Thus $S_{t}^{f_{1}} \geqslant T_{t}^{f_{1}} \geqslant e^{-c t} 1$.

Combining these results gives

$$
S_{t}^{f}=S_{t}^{f_{0}} S_{t}^{f_{1}} \geqslant S_{t}^{f_{0}} T_{t}^{f_{1}}>T_{t}^{f_{0}} T_{t}^{f_{1}}=T_{t}^{f} .
$$

Under more restrictive conditions one can obtain a converse to Proposition 5 and for this it is useful to generalize the irreducibility criteria for strict ordering given in II. The simplest connection of this type is the following. (Note that $L^{\infty}(X ; d \nu)$ is interpreted as a family of bounded multiplication operators on $\left.L^{p}(X ; d v).\right)$ 
LeMma 7. Let $S$ and $T$ be a $C_{0}$-semigroup on $\mathcal{X}=L^{p}(X ; d \nu)$ where $1<p<$ $\infty$.

If $S_{t}>0$ for some $t>0$ then $S_{t} \cup L^{\infty}(X ; d \nu)$ is irreducible.

If $S_{t}>T_{t}$ for some $t>0$ then $\left\{S_{t}-T_{t}\right\} \cup L^{\infty}(X ; d \nu)$ is irreducible.

Proof. Assume $\eta=S, \cup L^{\infty}(X ; d \nu)$ is not irreducible and choose a subspace $\mathscr{Y} \subset \mathcal{X}$ invariant under $\eta$. Next choose non-zero $\omega \in \mathcal{X}$, such that $\left.\omega\right|_{\mathscr{Q}}=0$ $|g|=f_{g} g,|\omega|=f_{\omega} \omega$ one has

$$
|\omega|\left(S_{\imath}|g|\right)=\omega\left(\bar{f}_{\omega} S_{\imath} f_{g} g\right)=0 .
$$

This is a contradiction.

The second statement is proved similarly.

To proceed we need to assume some analyticity.

LEMMA 8. Let $S$ be a holomorphic semigroup on $X=L^{p}(X ; d \nu)$ where $1<p<$ $\infty$ and suppose $S_{t} \geqslant 0$ for all $t>0$.

If $\omega \in \mathfrak{X}_{+}^{*}, g \in \mathfrak{X}_{+}$, then either $\omega\left(S_{t} g\right)>0$ for all $t>0$ or $\omega\left(S_{t} g\right)=0$ for all $t>0$.

Proof. Choose a sequence $t_{n}>0$ which converges to zero and is such that

$$
\left\|g-S_{t_{n}} g\right\|<2^{-n} \text {. }
$$

Then for $m \leqslant n$ define $g_{m, n}$ by

$$
g_{m, n}=g \wedge\left(\bigwedge_{m<k<n} S_{i_{k}} g\right)
$$

It follows that

$$
\begin{aligned}
\left\|g_{m, n}-g_{m, n+1}\right\| & <\left\|S_{t_{n}} g-S_{t_{n+1}} g\right\| \\
& <\left\|S_{t_{n}} g-g\right\|+\left\|g-S_{t_{n+1}} g\right\| \\
& <2^{-n+1} .
\end{aligned}
$$

Hence

$$
\begin{aligned}
\left\|g-g_{m, n}\right\| & <\left\|g-g_{m, m}\right\|+\sum_{k=m}^{n-1}\left\|g_{m, k}-g_{m, k+1}\right\| \\
& <2^{-m}+\sum_{k=m}^{n-1} 2^{-k+1} \\
& <2^{-m+3} .
\end{aligned}
$$

Now let $g_{m}=\lim _{n \rightarrow \infty} g_{m, n}$. One has $0 \leqslant g_{m}<g$ and $\left\|g-g_{m}\right\|<2^{-m+3}$. 
Now suppose that $\omega\left(S_{t_{0}} g\right)=0$ for some $t_{0}>0$. Since $g_{m}<g_{m, n}<S_{t_{n}} g$, $n>m$, and $S_{t} \geqslant 0$ one has

$$
0<\omega\left(S_{t} g_{m}\right)<\omega\left(S_{t+t_{n}} g\right), \quad n>m .
$$

Consequently $\omega\left(S_{t} g_{m}\right)=0$ for $t=t_{0}-t_{n}>0$ with $n>m$. Thus $t_{0}$ is an accumulation point of zero points of the holomorphic function $t \rightarrow \omega\left(S_{t} g_{m}\right)$. Hence $\omega\left(S_{t} g_{m}\right)=0$ for all $t>0$ and by limiting $\omega\left(S_{t} g\right)=0$ for all $t>0$.

This last lemma allows us to complete the characterization of strictly positive holomorphic semigroups.

THEOREM 9. Let $S$ be a holomorphic semigroup on $\mathcal{X}=L^{P}(X ; d \nu)$ where $1<p<\infty$ and suppose $S_{t} \geqslant 0$ for all $t>0$.

The following four conditions are equivalent

1. (1'.) $S_{t}>0$ for all $t>0$ (for one $\left.t>0\right)$.

2. (2'.) $\left\{S_{t}\right\}_{t>0} \cup L^{\infty}(X ; d \nu)$ is irreducible $\left(S_{t} \cup L^{\infty}(X ; d \nu)\right.$ is irreducible for some $t>0$ ).

Proof. Clearly $1 \Rightarrow 1^{\prime}$ and $2^{\prime} \Rightarrow 2$. Now $1^{\prime} \Rightarrow 2^{\prime}$ by Proposition 6 and hence it remains to prove that $2 \Rightarrow 1$.

Assume 1 is false; then there exist non-zero $\omega \in \mathscr{X}_{+}^{*}, g \in \mathfrak{X}_{+}$and a $t_{0}>0$ such that $\omega\left(S_{t_{0}} g\right)=0$. Hence $\omega\left(S_{t} g\right)=0$ for all $t>0$ by Lemma 8 . Next let $\mathcal{Y}$ be the closed linear span of

$$
\left\{f S_{t} g ; t>0, f \in L^{\infty}(X ; d v)\right\} .
$$

Now if $S_{f} \mathscr{Y} \subseteq \mathcal{O}$ and $\left.\omega\right|_{\mathscr{Y}}=0$ then $\mathscr{Y}$ is a non-trivial closed subspace of $\mathscr{X}$ invariant under $\left\{S_{t}\right\}_{t>0} \cup L^{\infty}(X ; d v)$. But these properties can be deduced by straightforward repetition of the argument given in II in the proof of Theorem 1.

REMARK. Theorem 1 of II contains other criteria which involve the resolvent of the generator of $S_{t}$. These criteria are also valid in the present context by similar arguments.

Next we derive an analogue of Theorem 4 of II which gives criteria for the strict ordering of two semigroups.

THEOREM 10. Let $S$ and $T$ be holomorphic semigroups on $\mathcal{X}=L^{P}(X ; d \mu)$ where $1<p<\infty$ and suppose that $S_{t} \geqslant T_{t} \geqslant 0$ for all $t>0$.

The following four conditions are equivalent

1. (1') $S_{t}>T_{t}$ for all $t>0$ (for one $\left.t>0\right)$.

2. $\left(2^{\prime}\right)\left\{S_{t}-T_{t}\right\}_{t>0} \cup L^{\infty}(X ; d \nu)$ is irreducible $\left(\left(S_{t}-T_{t}\right) \cup L^{\infty}(X ; d \nu)\right.$ is irreducible for one $t>0$ ). 
Proof. Clearly $1 \Rightarrow 1^{\prime}$ and $2^{\prime} \Rightarrow 2$. But $1^{\prime} \Rightarrow 2^{\prime}$ by Lemma 7 . Thus it remains to prove that $2 \Rightarrow 1$.

Suppose 1 is false then there is a $t_{0}>0$, and non-zero $\omega \in X_{+}^{*}, g \in \mathfrak{X}_{+}$, such that

$$
\omega\left(\left(S_{t_{0}}-T_{t_{0}}\right) g\right)=0 .
$$

But if $S_{t}=\exp \{-t H\}$ and $T_{t}=\exp \{-t K\}$ the operators $H S_{t}$ and $K T_{t}$ are bounded for all $t>0$. Therefore we introduce the two parameter family of bounded operators

$$
A(s, t)=S_{s} K T_{t}-H S_{s} T_{t}, \quad s, t>0,
$$

and remark that

$$
\begin{aligned}
A(s, t) & =\lim _{h \rightarrow 0+} h^{-1}\left\{\left(S_{s}-S_{s-h}\right) T_{t}-S_{s}\left(T_{t}-T_{t-h}\right)\right\} \\
& =\lim _{h \rightarrow 0+} h^{-1} S_{s-h}\left(S_{h}-T_{h}\right) T_{t-h}>0 .
\end{aligned}
$$

Moreover the Duhamel formula gives

$$
0=\omega\left(\left(S_{t_{0}}-T_{t_{0}}\right) g\right)=\lim _{\varepsilon \rightarrow 0} \int_{e}^{1-\varepsilon} d \lambda \omega\left(A\left(\lambda t_{0},(1-\lambda) t_{0}\right) g\right)
$$

and hence one must have

$$
\omega\left(A\left(\lambda t_{0},(1-\lambda) t_{0}\right) g\right)=0, \quad 0<\lambda<1 .
$$

Now for $t_{1}<\lambda t_{0}$ one has

$$
\omega\left(A\left(\lambda t_{0},(1-\lambda) t_{0}\right) g\right)=\omega\left(S_{t_{1}} h\right)
$$

where $h=A\left(\lambda t_{0}-t_{1},(1-\lambda) t_{0}\right) g \geqslant 0$. Thus by Lemma 8

$$
\omega\left(A\left(t,(1-\lambda) t_{0}\right) g\right)=0
$$

for all $t>0$ and $0<\lambda<1$. Hence by analyticity

$$
\omega(A(s, t) g)=0
$$

for all $s, t>0$.

Next let $\mathcal{Y}$ be the closed linear span of

$$
\left\{f A(s, t) g ; f \in L^{\infty}(X ; d v), s, t>0\right\}
$$

Clearly $L^{\infty}(X ; d \nu) \mathcal{Y} \subseteq \mathcal{Y}$ and we next argue, as in II, that $\mathscr{Y}$ is also invariant under $S$ and $T$. For example if

$$
\chi=\sum_{i=1}^{n} f_{i} A\left(s_{i}, t_{i}\right) g
$$

with $f_{i}$ real then

$$
\pm \chi \leqslant \sum_{i=1}^{n}\left\|f_{i}\right\|_{\infty} A\left(s_{i}, t_{i}\right) g
$$


and hence

$$
\left|S_{t} \chi\right| \leqslant \sum_{i=1}^{n}\left\|f_{i}\right\|_{\infty} A\left(s_{i}+t, t_{i}\right) g .
$$

Thus $S_{t} \chi \in \mathcal{Y}$. But if $\chi \in \mathcal{Y}$ then $|\chi| \in \mathcal{Y}$ because the subspace is invariant under $L^{\infty}(X ; d v)$. Hence the positive and negative parts $\chi_{ \pm}=(|\chi| \pm \chi) / 2$ are also in $\mathcal{Y}$ and

$$
0<T_{t} \chi_{ \pm}<S_{t} \chi_{ \pm} .
$$

The invariance of $\mathcal{Y}$ under $L^{\infty}(X ; d \nu)$ immediately implies that $T_{t} \chi_{ \pm} \in \mathcal{Y}$. Thus $T_{\mathfrak{t}} \mathscr{Y} \subseteq \mathscr{Y}$.

This establishes that $\mathcal{Y}$ is invariant under $\left\{S_{t}-T_{t}\right\}_{t>0} \cup L^{\infty}(X ; d \nu)$ and Condition 2 is false unless $\mathscr{Y}=\{0\}$ or $\mathscr{X}$. But $\mathscr{Y} \neq \mathscr{X}$ because $\left.\omega\right|_{\mathscr{Y}}=0$ and $\omega \neq 0$. Thus it remains to discuss the case $\mathscr{Y}=\{0\}$ and this discussion is a word for word repetition of that in II.

One introduces the closed linear span $\mathscr{D}$ of

$$
\left\{f T, g ; f \in L^{\infty}(X ; d \nu), t>0\right\}
$$

and argues from the assumption $\mathscr{Y}=\{0\}$ that $\mathscr{D}$ is invariant under $\eta=$ $\left\{S_{t}-T_{t}\right\}_{i>0} \cup L^{\infty}(X ; d \nu)$ and $\left.S_{t}\right|_{\mathscr{D}}=\left.T_{t}\right|_{\Phi}$. Thus $\left.\eta\right|_{\mathscr{D}}=\left.L^{\infty}(X ; d \nu)\right|_{\mathscr{D}}$ and condition 2 is again false.

Since this proof is essentially identical to the proof contained in II one has similar corollaries and one can also derive irreducibility criteria involving the resolvent.

Corollary 11. Let $S, T$, be two holomorphic semigroups on $\mathcal{X}=L^{p}(X ; d v)$, where $1<p<\infty$, and assume $S_{t} \geqslant T_{t} \geqslant 0$ for all $t>0$.

If $\mathscr{Y} \subset \mathcal{X}$ is invariant under $\left\{S_{t}\right\}_{r>0} \cup L^{\infty}(X ; d v)$ then it is invariant under $\left\{T_{t}\right\}_{t>0} \cup L^{\infty}(X ; d \nu)$.

If $S_{t}>0$ for some $t>0$ then $S_{t}>T_{t}$ for all $t>0$ or $S_{t}=T_{t}$ for all $t>0$.

Theorem 10 also allows us to prove a converse of Proposition 6.

Corollary 12. Let $S, T$, be two holomorphic semigroups of contractions on $X=L^{p}(X ; d \nu)$ where $1<p<\infty$ and assume $S_{t} \geqslant 0, T_{t} \geqslant 0$, for all $t>0$. Further let $f \in \mathfrak{N}$ be a function which is not constant on some non-empty sub-interval of $(0, \infty)$.

The following four conditions are equivalent

1. (1') $S_{t}>0$ for all $t>0$ (for some $\left.t>0\right)$.

2. (2') $S_{t}^{f}>0$ for all $t>0$ (for some $\left.t>0\right)$. 
Moreover if $S_{t} \geqslant T_{t}$ for all $t>0$ the following four conditions are equivalent

1. (1) $S_{t}>T_{t}$ for all $t>0$ (for some $\left.t>0\right)$.

2. (2') $S_{t}^{f}>T_{t}^{f}$ for all $t>0$ (for some $t>0$ ).

Proof. Consider the first four conditions. One has $1 \Leftrightarrow 1^{\prime}$ and $2 \Leftrightarrow 2^{\prime}$ by Theorem 9 and $1 \Rightarrow 2$ by Proposition 6. But 2 is equivalent to irreducibility of $\eta^{f}=\left\{S_{t}^{f}\right\}_{t>0} \cup L^{\infty}(X ; d \nu)$ by Theorem 9 and 1 is equivalent to irreducibility of $\eta=\left\{S_{t}\right\}_{t>0} \cup L^{\infty}(X ; d v)$. But from Definition 3

$$
S_{i}^{f}=\lim _{T \rightarrow \infty} S_{t}^{f_{T}}=\lim _{T \rightarrow \infty} \int_{0}^{\infty} d \mu_{f_{T}^{\prime}}(\tau) S_{\tau}
$$

and hence every subspace invariant under $\eta$ is automatically invariant under $\eta^{f}$. Thus if $\eta^{f}$ is irreducible $\eta$ is irreducible and $2 \Rightarrow 1$.

The proof of the second statement is similar but relies upon Proposition 6 and Theorem 10. Alternatively one can argue that $2 \Rightarrow 1$ by Corollary 11 . Explicitly $S_{t}^{f}>0$ implies $S_{t}>0$ by the above and hence $S_{t}^{f}>T_{t}^{f} \geqslant 0$ implies $S_{t}>T_{t} \geqslant 0$ or $S_{t}=T_{t}$ by Corollary 11. But if $S_{t}=T_{t}$ for all $t>0$ then $S_{t}^{f}=T_{t}^{f}$ which is a contradiction.

REMARK. Corollary 12 is also valid for $p=1$ and $f \in \mathscr{T}_{0}$.

\section{Conclusion}

The foregoing results can be extended in a variety of ways. If one only examines functions $f \in \mathfrak{R}_{0}$ one can analyze order properties on general Banach spaces or on function spaces other than the $L^{p}$-spaces. It is also possible to obtain most of our results for $C_{0}^{*}$-semigroups. Recall that if the Banach space $\mathscr{X}$ is the dual of a Banach space $X_{*}$ and $S$ is a $C_{0}$-semigroup on $X_{*}$ then its dual $S^{*}$ on $\mathcal{X}$ is defined to be a $C_{0}^{*}$-semigroup and the dual semigroup $S^{*}$ is defined to be a holomorphic semigroup if the functions $t \rightarrow \omega\left(S_{t}^{*} g\right)$, where $\omega \in \mathcal{X}$, and $g \in \mathcal{X}$, have the appropriate analyticity properties. This type of semigroup is much more appropriate to the discussion of $L^{\infty}$.

Finally one can use the definition of $S^{f}$ and $T^{f}$ given in Section 2 to demonstrate stability properties of the domination relation discussed by Hess, Schrader, Uhlenbrock (Hess, Schrader, Uhlenbrock (1977)) and Simon (Simon (1979)). For example if $X$ is a function space then $S$ is said to dominate $T$ if

$$
S_{t}|g|>\left|T_{t} g\right|
$$

for all $t>0$. But then

$$
S_{t}^{f}|g|=\int d \mu_{f}^{t}(\tau) S_{\tau}|g|>\int d \mu_{f}^{t}(\tau)\left|T_{\tau} g\right|>\left|T_{\imath}^{f} g\right|
$$

for $f \in \mathfrak{T}_{0}$ and by limiting for $f \in \mathfrak{R}$, where appropriate. 
Acknowledgement. The authors are grateful to Ola Bratteli for a number of helpful discussions.

\section{Appendix}

In this appendix we derive two results which are relevant to the foregoing analysis. The first concerns continuity properties of semigroups acting on $L^{\infty}(X ; d \nu)$ and the second extends the monotone convergence theorems established in II for $L^{2}(X ; d \nu)$ to $L^{p}(X ; d \nu)$ where $1<p<\infty$.

Proposition A1. Let $\left(\theta_{n}\right)$ be a sequence of positivity preserving continuous linear maps on the Banach space $L^{\infty}(x ; d \nu)$. If $\left\|\theta_{n}(\psi)-\psi\right\|_{\infty} \rightarrow 0$ as $n \rightarrow \infty$ for every $\psi \in L^{\infty}$, then $\left\|\theta_{n}-1\right\| \rightarrow 0$ as $n \rightarrow \infty$.

Proof. Suppose that $\left\|\theta_{n}-1\right\| \nrightarrow 0$. Then by taking a subsequence if necessary we may assume that for some $\varepsilon>0$

$$
\left\|\theta_{n}-1\right\|>\varepsilon, \quad n=1,2, \ldots
$$

Hence there is a sequence of vectors $\psi_{n}$ in $L^{\infty}$ such that

$$
0<\psi_{n}(x)<1, \quad \text { a.a. } x \in X, \quad\left\|\theta_{n}\left(\psi_{n}\right)-\psi_{n}\right\|_{\infty}>\varepsilon / 4 \text {. }
$$

By replacing $\psi_{n}$ by $1-\psi_{n}$ if necessary we may assume that

$$
\text { ess.sup }\left\{\psi_{n}(x)-\theta_{n} \psi_{n}(x)\right\}>\varepsilon / 5
$$

for large $n$ (such that $\left\|1-\theta_{n} 1\right\|_{\infty}<\varepsilon / 20$ ). Let

$$
X_{n}=\left\{x \in X ; \psi_{n}(x)-\theta_{n} \psi_{n}(x)>\varepsilon / 5\right\} .
$$

We assert that there are a subsequence $\left(n_{k}\right)$ of $(n)$ and non-null measurable sets $\Omega_{k}$ such that $\Omega_{k} \subset X_{n_{k}}$ and $\left(\Omega_{k}\right)$ are mutually disjoint. For $\varphi_{k} \equiv \psi_{n_{k}} \chi_{\Omega_{k}}$ and $x \in \Omega_{k}$ we have

$$
\varphi_{k}(x)-\theta_{n_{k}} \varphi_{k}(x)>\psi_{n_{k}}(x)-\theta_{n_{k}} \psi_{m_{k}}(x)>\varepsilon / 5 .
$$

Because $\theta_{n_{k}}$ is positivity-preserving and $\varphi_{k}, \psi_{n_{k}}$ are equal on $\Omega_{k},\left\|\varphi_{k}-\theta_{n_{k}} \varphi_{k}\right\|_{\infty}>$ e $/ 5$.

The rest of the proof now follows from the lemma of Elliott (Elliott (1972)) which is a rephrasing of a well-known lemma of Phillips' (Phillips (1940)).

It remains to prove the existence of the $\Omega_{n}$.

First assume that

$$
\overline{\lim } X_{k} \equiv \bigcap_{n} \bigcup_{k>n} X_{k}
$$

is non-null. Then $\nu \mid \overline{\lim } X_{k}$ is completely non-atomic, since, by strong convergence of $\theta_{n}$, any atom is eventually not contained in $X_{n}$ for large $n$. Hence we 
can find an infinite family $\left(Y_{k}\right)$ of mutally disjoint non-null subsets such that

$$
\varlimsup X_{k}=\bigcup_{k=1}^{\infty} Y_{k} \text {. }
$$

We define inductively $n_{k}$ for each $k$ such that $n_{k}>n_{k-1}$ and $\nu\left(Y_{k} \cap X_{n_{k}}\right)>0$ (let $n_{0}=0$ ). Setting $\Omega_{k}=Y_{k} \cap X_{n_{k}}$, we have the assertion.

If $\lim X_{k}$ is null, let

$$
\Omega_{k}=X_{k} \backslash \bigcup_{n>k} X_{n} .
$$

Clearly $\left(\Omega_{k}\right)$ are mutually disjoint and there are infinitely many non-null $\Omega_{k}$. This establishes the assertion.

COROLlaRY A2. Each $C_{0}$-semigroup of positivity preserving maps on $L^{\infty}(X ; d \nu)$ is uniformly continuous.

Remark. It is possible that Corollary A2 is valid without the positivity-preserving assumptions. We do not know of any examples of $C_{0}$-semigroups on $L^{\infty}(X ; d \nu)$ which are not uniformly continuous.

Now we turn to the examination of monotone convergence properties of positivity preserving operators. These can be deduced from monotone convergence results for weakly complete Banach spaces which have been studied by various authors (see, for example, Krasnoselski (1964), Karlin (1959)). To summarize these results we first need some general terminology concerning ordered Banach spaces.

Let $\mathcal{X}$ be a real Banach space. A cone $\mathscr{K}$ in $\mathcal{X}$ is a subset such that $\mathscr{K}+\mathscr{K} \subset \mathscr{K}, \lambda \mathscr{K} \subseteq \mathscr{K}$ for all $\lambda \geqslant 0$, and $\mathscr{K} \cap-\mathscr{K}=\{0\}$. Each such cone defines a partial order on $\mathfrak{X}$ by $A \geqslant 0$ if $A \in \mathscr{K}$ and $A>B>0$ if $A-B$, $B \in \mathscr{K}$. The cone $\mathscr{K}$ is said to generate $\mathscr{X}$ if $\mathscr{X}=\mathscr{K}-\mathscr{K}$ and $\mathscr{K}$ is said to be normal if there is a constant $\gamma>0$ such that $\|A+B\|>\gamma \max \{\|A\|,\|B\|\}$ for all $A, B \in \mathcal{K}$.

Proposition A3. Let $\mathscr{K}$ be a closed cone in a Banach space $\mathcal{X}$ and assume the unit ball $\mathfrak{X}_{1}$ of $\mathfrak{X}$ is sequentially weakly complete.

The following conditions are equivalent

1. $\mathscr{K}$ is normal.

2. Each monotonically increasing order-bounded sequence in $\mathscr{K}$ is norm-convergent.

3. Each monotonically increasing norm-bounded sequence in $\mathscr{K}$ is norm convergent. 
The proof of $3 \Leftrightarrow 2 \Rightarrow 1$ is given by Krasnoselski (Krasnoselski (1964)). A proof of $1 \Rightarrow 2$ was given by Karlin (Karlin (1959)).

Next we define a bounded operator $S$ on $\mathcal{X}$ to be $\mathcal{K}$-positivity preserving if $S \mathscr{K} \subseteq \mathscr{K}$ and we set $S \geqslant 0$. Similarly we write $S \geqslant T \geqslant 0$ if $S-T$ and $T$ are $\mathcal{K}$-positivity preserving, that is $S \geqslant T \geqslant 0$ if, and only if, $S A>T A>0$ for all $A \in \mathscr{K}$. One can now deduce the following result from Proposition A3.

Proposition A4. Let $\mathscr{K}$ be a closed normal cone which generates the Banach space $\mathcal{X}$ and assume that the unit ball $\mathcal{X}_{1}$ of $\mathfrak{X}$ is sequentially weakly complete. Further let $S_{n}$ be a norm-bounded, or order bounded, sequence of $\mathfrak{K}_{\text {-positivity }}$ preserving operators which is monotonically increasing with respect to the partial order $\geqslant$.

It follows that $S_{n}$ converges strongly to a $\mathcal{K}_{\text {-positivity preserving operator } S \text { and }}$ $S_{n} \leqslant S$.

REMARKs. 1. The conclusions of both propositions extend from sequences to nets if $\mathscr{X}_{1}$ is weakly complete.

2. If $\mathcal{X}$ is reflexive then $\mathcal{X}_{1}$ is weakly complete. In particular this is the case for $\mathcal{X}=L^{p}(X ; d \nu)$ with $1<p<\infty$.

3. The unit ball of $L^{1}(X ; d \nu)$ is sequentially weakly complete but in general it is not weakly complete.

4. In $X=L^{\infty}(\mathbf{R}, d x)$ the characteristic functions $\chi_{n}$ of the interval $[-n, n]$ form a positive monotonically increasing sequence which is norm-bounded but not norm-convergent. Thus monotone convergence theorems are not generally valid for $L^{\infty}$-spaces.

5. Analogues of both propositions can be proved for monotonically decreasing sequences.

\section{References}

C. Berg and G. Forst (1975), Potential theory on locally compact Abelian groups (Springer-Verlag).

A. Beurling and J. Deny (1958), 'Espaces de Dirichlet', Acta Math. 99, 203-224.

S. Bochner (1955), Harmonic analysis and the theory of probability, (Berkeley).

O. Bratteli, A. Kishimoto and D. W. Robinson (1980), 'Positivity and monotonicity properties of $C_{0}$-semigroups I', Comm. Math. Phys. 75, 67-84.

G. A. Elliott, 'Convergence of automorphisms in certain *-algebras', J. Functional Analysis 11, 204-206.

H. Hess, R. Schrader and D. W. Uhlenbrock (1977), 'Domination of semigroups and generalization of Kato's inequality', Duke Math. J. 44, 893-904.

E. Hille and R. S. Phillips (1957), Functional analysis and semi-groups, (Amer. Math. Soc. Colloq. Publ. 31, Providence).

S. Karlin (1959), 'Positive operators', J. Math. Mech. 8, 907-937. 
A. Kishimoto and D. W. Robinson (1980), 'Positivity and monotonicity properties of $C_{0^{-}}$ semigroups', Comm. Math. Phys. 75, 85-101.

M. A. Krasnoselski (1964), Positive solutions of operator equations, (Noordhoff).

E. Nelson (1958), 'A functional calculus using singular Laplace integrals', Trans. Amer. Math. Soc. 88, 400-413.

R. S. Phillips (1952), 'On the generation of semigroups of linear operators', Pacific J. Math. 2, 343-369.

R. S. Phillips, 'On linear transformation', Trans. Amer. Math. Soc. 48, 516-541.

I. J. Schoenberg (1938), 'Metric spaces and completely monotone functions', Ann. Math. 39, 811-841.

B. Simon (1979), 'Kato's inequality and the comparison of semigroups', J. Functional Analysis 32, 97-101.

Department of Pure Mathematics

University of New South Wales

Kensington 2033

Australia 